\title{
Accurate Potential Energy Surfaces and Beyond: Chemical Reactivity, Binding, Long-Range Interactions, and Spectroscopy
}

\author{
Laimutis Bytautas, ${ }^{1}$ Joel M. Bowman, ${ }^{2}$ Xinchuan Huang, ${ }^{3}$ and António J. C. Varandas ${ }^{4}$ \\ ${ }^{1}$ Department of Chemistry, Rice University, Houston, TX 77005, USA \\ ${ }^{2}$ Cherry L. Emerson Center for Scientific Computation and Department of Chemistry, Emory University, Atlanta, GA 30322, USA \\ ${ }^{3}$ SETI Institute and NASA Ames Research Center, MS 245-6, Moffett Field, CA 94035, USA \\ ${ }^{4}$ Departamento de Quimica, Universidade de Coimbra, 3004535 Coimbra, Portugal \\ Correspondence should be addressed to Laimutis Bytautas, laimutis.bytautas@rice.edu
}

Received 13 February 2012; Accepted 13 February 2012

Copyright (c) 2012 Laimutis Bytautas et al. This is an open access article distributed under the Creative Commons Attribution License, which permits unrestricted use, distribution, and reproduction in any medium, provided the original work is properly cited.

\section{Background}

Beginning with the seminal paper of Born and Oppenheimer (BO) [1] in 1927, the concept of the potential energy surface (PES) plays a critical role in the description, simulation, and modeling of molecular systems. It provides the basis [2] for understanding the processes associated with the nuclear motions in molecules. By going beyond the characteristic stationary points and barriers, full dimensional, accurate potential energy surfaces have a very broad range of applications in many areas of physical chemistry; for example, they provide insight into structure, reactivity, and spectroscopy of molecules. While the majority of stable structures on PES are associated with the covalent or ionic bonding [3], the regions of PES dominated by van der Waals interactions are essential for low-temperature phenomena and molecular stacking which are critical for understanding biomolecular structures involving DNA and RNA molecules [4]. Furthermore, the advances in "cold chemistry" [5] make it possible to test the theoretical predictions (see, e.g., [6]) involving very small barriers of $<15 \mathrm{~K}$. At such low temperatures the quantum effects, for example, tunneling, play a significant role in chemical reactivity. For instance, the large de Broglie wavelength of ultracold molecules entirely changes the nature of reaction dynamics [7], and energy barriers on the PES play a different role because, in this regime, quantum tunneling becomes the dominant reaction pathway [8]. In addition, our understanding of potential energy surfaces has benefitted greatly from the ability of experimentalists to study chemical reactions and "observe" transition states in real time using the transition-state spectroscopy [9].
Due to the recent advances in ab initio method development [10] primarily focusing on the solution of the nonrelativistic Schrödinger equation, the theoretical data representing PESs is of higher quality and the cost and timing for such calculations is considerably improved. Furthermore, the relativistic corrections [11] can be significant and should be included if high accuracy of PESs is needed. For instance, the inclusion of the spin-orbit coupling effect (relativistic phenomenon) may turn a crossing of two potential energy curves into an avoided crossing [12]. Finally, given the raw $a b$ initio data, efficient fitting techniques [13] are capable of generating excellent analytical representations of potential energy surfaces. One example is the functional representation of a PES using the double many-body expansion method [14]. Another important class of PESs corresponds to the ones that are constructed to be explicitly invariant with respect to all permutations of equivalent atoms [15, 16]. It is well recognized that one of the most stringent criteria of the quality of the PES is its ability to reproduce the experimental rotational-vibrational spectrum with the "near-spectroscopic" accuracy [12] of about $10 \mathrm{~cm}^{-1}$ or better [17]. Occasionally, the empirical refinements for $a b$ initio PESs are used in order to achieve a very close agreement $\left(<0.1 \mathrm{~cm}^{-1}\right)$ with experiment for rovibrational transitions [18].

In many cases the $\mathrm{BO}$ approximation is valid to a high degree, and a single PES is sufficient to describe the motion of nuclei. However, when several electronic states get close in energy, the coupling between different potential energy surfaces becomes significant. The crossings of several PESs 
(e.g., conical intersections) or avoided crossings require a more refined treatment of nuclear dynamics which extends nuclear motion to more than one BO surface [19]. The conical intersections or "seams" $[20,21]$ play an important role in photochemistry, for example, contributing to the photostability of DNA and participating in the isomerization process of cofactor retinal that initiates visual reception.

\section{The Present Issue}

The current special issue (ten papers: six reviews and four research articles) represents an excellent collection of papers focusing on the various aspects of potential energy surfaces.

The paper "Constructing potential energy surfaces for polyatomic systems: recent progress and new problems" by J. Espinosa-Garcia, M. Monge-Palacios, and J. C. Corchado provides a comprehensive description of different methods for constructing PESs based on electronic structure calculations, and their performance is evaluated by calculating properties associated with chemical reaction dynamics. The authors conclude that at the present stage the field of small systems has become mature; however the realm of larger polyatomic systems requires considerable improvement, especially when dealing with relativistic (e.g., spin-orbit) effects.

M. Ayouz and D. Babikov in their research paper "Improved potential energy surface of ozone constructed using the fitting by permutationally invariant polynomial function" illustrate the advantages of their method by studying the formation of ozone at thermal energies and its spectroscopy near the dissociation limit. The authors demonstrate that the approach of fitting the ab initio data by permutationally invariant polynomial functions allows the construction of accurate global PESs for symmetric molecules using only relatively small number of points.

The research paper " $A b$ initio potential energy surfaces for both the ground $\left(\widetilde{X}^{1} A^{\prime}\right)$ and excited $\left(\widetilde{A}^{1} A^{\prime \prime}\right)$ electronic states of $\mathrm{HSiBr}$ and the absorption and emission spectra of $\mathrm{HSiBr} / \mathrm{DSiBr}$ " by A. Li, S. Lin, and D. Xie illustrate the high quality of the PESs by calculating the vibrational energy levels for the ground and excited states of these challenging systems. The latter were found to be in good agreement with the available experimental band origins.

The surface diffusion of adsorbed atoms and molecules plays a significant role in various surface dynamical processes, e.g., in heterogeneous chemical reactions and formation of self-assembled structures. N. Tsukahara and J. Yoshinobu in the paper "Potential energy surface of NO on $\operatorname{Pt}(997)$ : adsorbed states and surface diffusion" elucidate the PES by investigating the adsorption states and diffusion processes of $\mathrm{NO}$ on $\mathrm{Pt}(997)$ using infrared absorption spectroscopy (IRAS) and scanning tunneling microscopy (STM).

The classical transition state theory (TST) has been very popular over the years for calculating the reaction rate constants, and it has been quite successful in the high-temperature regime. However, at low temperatures, especially involving light nuclei, the quantum effects become significant. Quantum instanton (QI) approximation has been introduced as one way to quantize the TST by naturally incorporating the quantum effects, like, for example, tunneling. For this reason, the quantum instanton approximation has received much attention for estimating the chemical reaction rate constants using full-dimensional potential energy surfaces. The QI can be applied to quite complex molecular systems via well-established imaginary time path integral techniques as described by Y. Zhao and W. Wang in their paper "Quantum instanton evaluations of the thermal rate constants for complex systems." The authors demonstrate the utility of the QI method by applying it to such complex processes like $\mathrm{H}$ diffusion on $\mathrm{Ni}(100)$ surface, and surfacesubsurface transport and interior migration for $\mathrm{H} / \mathrm{Ni}$.

The hydrogen bonding plays a significant role in living organisms, for example, between DNA or RNA bases. The cooperativity of hydrogen bonding and the substituent effects are very important in stabilizing biomolecules. In this issue, A. Ebrahimi, S. M. Habibi-Khorassani, F. B. Akher, and A. Farrokhzadeh in the research article "The N . . HF interactions in the X-pyridazine $\cdots(\mathrm{HF})_{2}$ complexes: substituent effects and energy components" investigate the strength of the hydrogen bonding between $\mathrm{X}$-pyridazine ( $\mathrm{N}$-sites) and HF molecules depending on the substituent. The authors find that in all complexes the binding energies $(\mathrm{N} \cdots \mathrm{HF})$ increase for electron-donating substituents and decrease for electron-withdrawing substituents. Also a negative cooperativity is observed for two hydrogen bond interactions.

In order to understand the entire photochemical reaction process of a given system, it is necessary to explore several PESs that represent ground and excited states. Within the Franck-Condon (FC) approximation, a reaction starts from the FC point on an excited-state surface. Then, depending on the topography and the available excess energy, the system may select either adiabatic or nonadiabatic pathway. In the adiabatic case, the reacting system moves on the excited PES surpassing transition state to yield products. In the nonadiabatic case, the system undergoes a nonadiabatic transition (or several transitions cascading through a number of PESs) and then moves on the PES of the lower state. In the later case, the seams of intersection between two PESs play an important role. S. Maeda, K. Ohno, and K. Morokuma in the review paper "Exploring multiple potential energy surfaces: photochemistry of small carbonyl compounds" describe the global reaction route mapping (GRRM) method to explore the critical regions such as transition states, conical intersections, intersection seams, and minima associated with multiple PESs. The authors illustrate the efficiency of the method by documenting new nonadiabatic pathways in the photochemistry of formaldehyde and acetone.

Enzymatic reactions exploring catalytic properties of enzymes represent a very intense area of chemical research. Many species encountered in enzymatic reactions, especially metalloenzymes, may have more than one electronic state lying close in energy. It is possible that as reaction progresses, the relative energy separation between states may vary, and in particular the energetic ordering of these states may change during the reaction. Such mechanisms may include 
cases where the "switching" involves PESs of different spin multiplicities. The likelihood of such multistate reactivity may have important consequences in enzymatic reactions. E. A. C. Bushnell, W. Huang, and J. Gauld in the paper "Applications of potential energy surfaces in the study of enzymatic reactions" explore several states of different spin multiplicities in the catalytic activation of $\mathrm{O}_{2}$ by alpha-ketoglutarate-dependent dioxygenase (AlkB).

The photochemical studies where the metal atom is photoexcited into an upper state and then reacts with a given molecule (e.g., methane) are quite informative since the reaction pathway and probability strongly depend on the initial electronic state of an atom. Furthermore, the reaction pathway frequently encounters several potential energy surfaces (with various multiplicities) before the final products are formed. In this issue, O. Novaro, M. del Alba Pacheco-Blas, and J. H. Pacheco-Sánchez in the paper "Potential energy surfaces for reactions of $\mathrm{X}$ metal atoms $\left(\mathrm{X}=\mathrm{Cu}, \mathrm{Zn}, \mathrm{Cd}, \mathrm{Ga}, \mathrm{Al}, \mathrm{Au}\right.$, or $\mathrm{Hg}$ ) with $\mathrm{YH}_{4}$ molecules $(\mathrm{Y}=\mathrm{C}, \mathrm{Si}$, or $\mathrm{Ge})$ and transition probabilities at avoided crossings in some cases" discuss the possible mechanisms for the reaction of the metal atom with the gas molecules. In the cases above, the authors show that reaction pathways encounter many surfaces, and the transition probabilities for going from one surface to another are calculated using timedependent Landau-Zener theory. The results indicate a good agreement with the experimental data whenever they are available.

An example of the $\mathrm{BO}$ approximation breakdown is the Renner-Teller effect which is due to the fact that at linear geometries many of the electronic states in molecules are twofold degenerate. This results in the coupling of the electronic motion with the nuclear motion giving rise to the so-called rovibronic coupling. H. Ma, C. Zhang, Z. Zhang, X. Liu, and W. Bian in their research article "New $a b$ initio potential energy surfaces for the Renner-Teller coupled $1^{1} A^{\prime}$ and $1^{1} A^{\prime \prime}$ states of $\mathrm{CH}_{2}$ " generate the analytical representations of the PESs using a dual-level (the lower level and the higher level which also include core and core-valence correlation effects) strategy with the inclusion of the RennerTeller terms. The authors use these PESs for the quantum dynamical calculations and demonstrate that the calculated vibronic energy levels for the two singlet electronic states are in excellent agreement with experiment.

Finally, we would like to thank all the authors for providing the high-quality papers and also all the reviewers who donated their valuable time to this special issue.

Laimutis Bytautas Joel M. Bowman Xinchuan Huang António J. C. Varandas

\section{References}

[1] M. Born and J. R. Oppenheimer, "Zur quantentheorie kontinuierlicher spektren," Annalen der Physik, vol. 41, no. 8-9, pp. 457-484, 1927.
[2] J. N. Murrell, S. Carter, S. C. Farantos, P. Huxley, and A. J. C. Varandas, Molecular Potential Energy Functions, John Wiley \& Sons, Chichester, UK, 1984.

[3] P. Ball, "Beyond the bond," Nature, vol. 469, no. 7328, pp. 2628,2011

[4] K. E. Riley, M. Pitoňák, P. Jurečka, and P. Hobza, "Stabilization and structure calculations for noncovalent interactions in extended molecular systems based on wave function and density functional theories," Chemical Reviews, vol. 110, no. 9, pp. 5023-5063, 2010.

[5] D. Herschbach, "Molecular collisions, from warm to ultracold," Faraday Discussions, vol. 142, pp. 9-23, 2009.

[6] L. Bytautas and K. Ruedenberg, "Ab initio potential energy curve of $\mathrm{F}_{2}$. IV. Transition from the covalent to the van der Waals region: competition between multipolar and correlation forces," Journal of Chemical Physics, vol. 130, no. 20, Article ID 204101, 2009.

[7] R. V. Krems, "Cold controlled chemistry," Physical Chemistry Chemical Physics, vol. 10, no. 28, pp. 4079-4092, 2008.

[8] P. R. Schreiner, H. P. Reisenauer, D. Ley, D. Gerbig, C.-H. Wu, and W. D. Allen, "Methylhydroxycarbene: tunneling control of a chemical reaction," Science, vol. 332, no. 6035, pp. 1300$1303,2011$.

[9] A. H. Zewail, "Femtochemistry: recent progress in studies of dynamics and control of reactions and their transition states," Journal of Physical Chemistry, vol. 100, no. 31, pp. 1270112724, 1996.

[10] C. D. Sherrill, "Frontiers in electronic structure theory," Journal of Chemical Physics, vol. 132, no. 11, Article ID 110902, 2010.

[11] P. Pyykkö, "Relativistic effects in structural chemistry," Chemical Reviews, vol. 88, no. 3, pp. 563-594, 1988.

[12] L. Bytautas, N. Matsunaga, and K. Ruedenberg, "Accurate $a b$ initio potential energy curve of $\mathrm{O}_{2}$ II. Core-valence correlations, relativistic contributions, and vibration-rotation spectrum," Journal of Chemical Physics, vol. 132, no. 7, Article ID 074307, 2010.

[13] G. C. Schatz, "The analytical representation of electronic potential-energy surfaces," Reviews of Modern Physics, vol. 61, no. 3, pp. 669-688, 1989.

[14] A. J. C. Varandas, "Intermolecular and intramolecular potentials: topographical aspects, calculation, and functional representation via a double many-body expansion method," Advances in Chemical Physics, vol. 74, pp. 255-338, 1988.

[15] B. J. Braams and J. M. Bowman, "Permutationally invariant potential energy surfaces in high dimensionality," International Reviews in Physical Chemistry, vol. 28, no. 4, pp. 577606, 2009.

[16] J. M. Bowman, B. J. Braams, S. Carter et al., "Ab-initiobased potential energy surfaces for complex molecules and molecular complexes," Journal of Physical Chemistry Letters, vol. 1, no. 12, pp. 1866-1874, 2010.

[17] O. L. Polyansky, A. G. Császár, S. V. Shirin et al., "Highaccuracy $a b$ initio rotation-vibration transitions for water," Science, vol. 299, no. 5606, pp. 539-542, 2003.

[18] X. Huang, D. W. Schwenke, and T. J. Lee, "Rovibrational spectra of ammonia. I. Unprecedented accuracy of a potential energy surface used with nonadiabatic corrections," Journal of Chemical Physics, vol. 134, no. 4, Article ID 044320, 15 pages, 2011.

[19] G. A. Worth and L. S. Cederbaum, "Beyond born-oppenheimer: molecular dynamics through a conical intersection," Annual Review of Physical Chemistry, vol. 55, pp. 127-158, 2004. 
[20] D. R. Yarkony, "Conical intersections: the new conventional wisdom," Journal of Physical Chemistry A, vol. 105, no. 26, pp. 6277-6293, 2001.

[21] T. J. Martinez, "Physical chemistry: seaming is believing," Nature, vol. 467, no. 7314, pp. 412-413, 2010. 


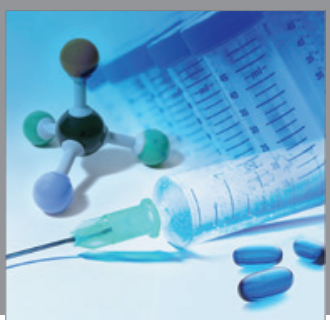

International Journal of

Medicinal Chemistry

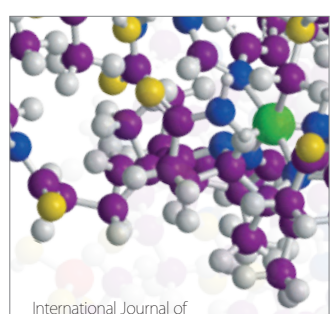

Carbohydrate Chemistry

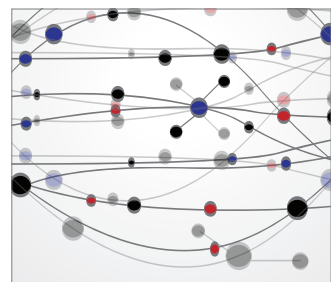

The Scientific World Journal
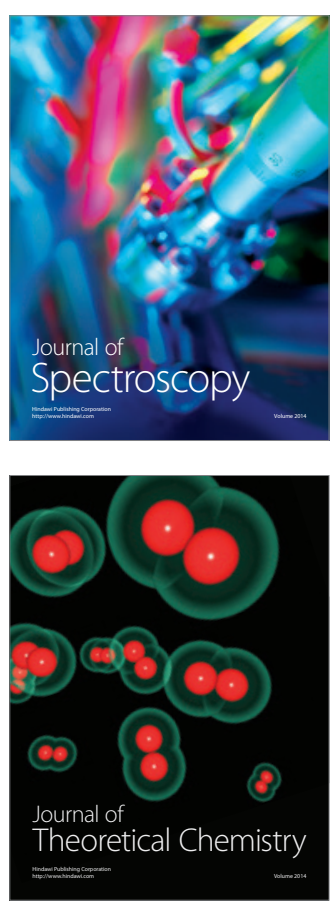
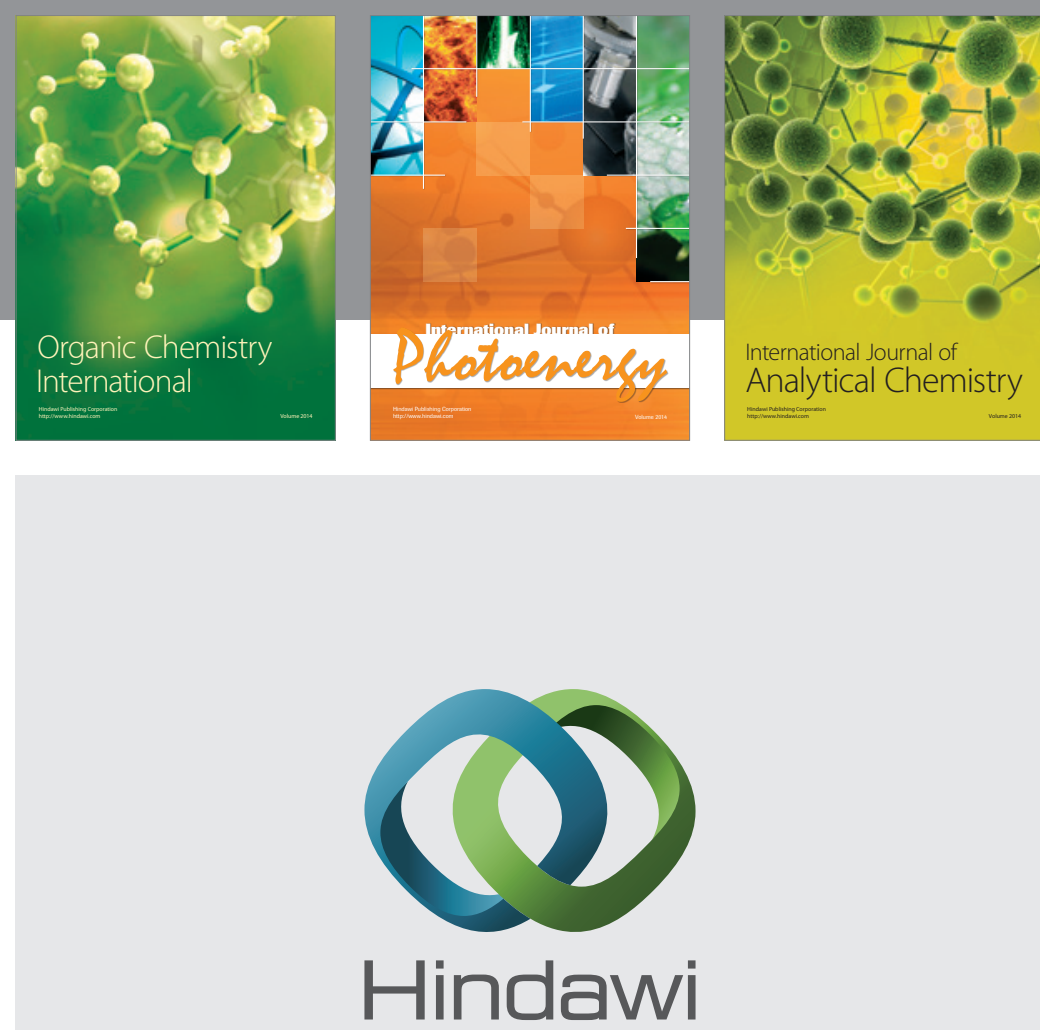

Submit your manuscripts at

http://www.hindawi.com
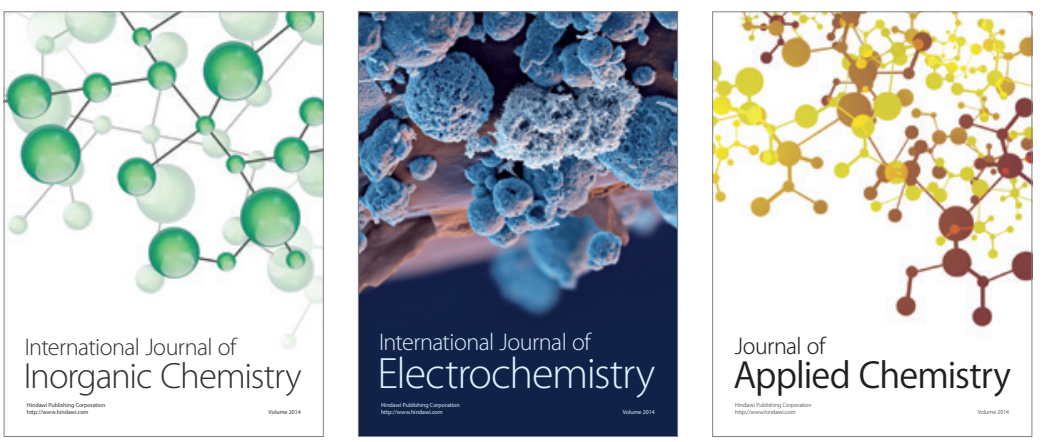

Journal of

Applied Chemistry
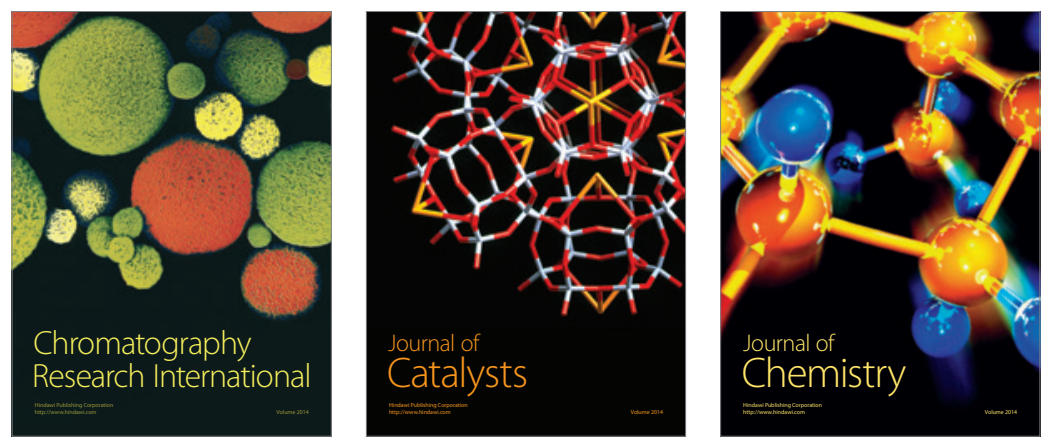
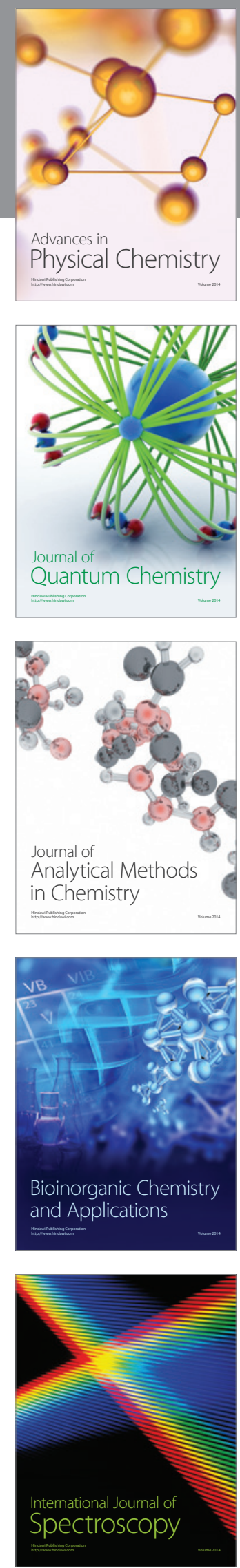